\title{
Network Theory of Living Cell Clusters and Rheological Applications at Nano-Level
}

\author{
M.B. Plavsic*, I. Pajic-Lijakovic, M.M. Plavsic, B. Bugarski \\ Faculty of Technology and Metallurgy, Belgrade University, Belgrade, Serbia
}

\begin{abstract}
The system of living cells closed in a polymer matrix and self-organized into clusters is considered, extending free volume concept developed for complex system interactions quantification in statistical mechanics of jammed state of matter. Possibility of extension of Edwards concept of compactivity and angorisity developed for hard irregular grains with friction to living cell systems, is considered. Existences of scaling laws for cell colony grow, related to their self assembling and response to polymer hydrogel micro-environment constrains, is analyzed as function of rate of cluster density increase. Based on the theory proposed are developed relations, connecting cluster properties that are difficult to measure, to data from standard cell cultivation experiments. The model also provides possibilities of incorporation data on single cell behavior, available from modern nano- rheology measurements, into cluster
\end{abstract}

PACS: 05.20.--y, 36.20.-f, 64.75.Yz, 87.18.--h

\section{Introduction}

Interactions of a cell with neighboring cells in cellcolony or tissue, as well as interactions with extracellular matrix (ECM) trigger numerous responses that have essential roles in cell's life including cell arrangement in clusters, food transport and cell migration but also its proliferation, differentiation, growth and death, especially apoptosis [1].The most essential part of ECM for functions described is polymer network constituted from polysaccharide chains and fibrous proteins that fill the interstitial space between cells and incorporating body liquids build gels. Also cytoskeleton is a polymer network but of different kind. Cytoskeleton of eukaryotic cells contain three main kinds of filament: microfilament (made of actin polymeric chains), intermediate filaments (made of vimentins, keratins etc.) and microtubules (tubuline nano-networks). It may be useful to think of cytoskeleton in general as dynamic network with microand intermediate- filaments as cables and microtubules as cellular support beams. Here is especially important that intermediate filaments mainly organize the internal tridimensional cell structure and participate in cell-cell, cell-ECM or cell-support-matrix junctions.

It follows that a number of essential features of such complex systems as tissues (i.e. cells together with ECM) or cell colonies with supporting gels (in bioengineering cell immobilizing devices e.g. alginate gel beads) can be considered as "united" polymeric network, interpenetrating the whole system as described above. It helps not only to understanding the architecture of the sys-

* corresponding author; e-mail: plavsic@tmf.bg.ac.rs tem and its mechanical properties but also numerous cell functions. Cytoskeleton filaments anchor organelles and serve as structural components of nuclear lamina and sarcomers. So forces transferred from ECM or arrived in the course of adhesion are related to information transfer through the united network, taking part in distinction of "self" and "foreign" in immune response and gene activation, formation of tissue and healing of wounds. Moreover, last years grows the evidence of cell-cell communication of unicellular organisms tending to organize into complex communities that behave analogous to multicellular organisms (organize united self-defense, hunting for prey etc.) and making such intercellular network connections as described. The silent aspect of such organization is cell-cell signaling mechanism that can be understood also as a kind of informational network been dual to described polymer (connectivity) networks [2-4].

Even more exciting experimental evidence are recent results on formation and function of such networks in the case of dynamic onset of Dictyostelium discoideum from a collision of unicellular organisms to a multi-cellular slug and finally to a fruiting body as multi-cellular amoeba organism [5]. The cell aggregation starts with stochastic pulsing of individual cells with release of signaling molecules. But, at some density of cell "crowding" and density of signaling molecules in interstitial space starts synchronization of such cell oscillations and the new kind of behavior, organization and "wholeness" emerges what is characteristics of systems considered in complexity science approach of modern physics. Although in many papers have been investigated constitution and biochemical properties of particular components of the cell clusters and supporting gels or ECM, the whole system described 
as a connectivity network is not considered. In this contribution we propose a method that resolves the complex structures described, in particular with stochastic behavior of individual cells, to some basic physical properties that can be elaborated in terms of jammed state statistical mechanics. Relations obtained provide possibilities for interpretation of cluster rheological properties at nano-level and can be used in further research of cell cluster organization and growth.

\section{Theory}

It has been recognized for pretty long time that cellular systems (in particular polymer foams) as well as granular systems with large number of particles (or cells) evolve into steady state of similar structures, irrespective of the specific dynamics and of the length scale over which the dynamics take place [6]. This prompts the use of statistical mechanical methods to define global characteristics (in de Genne's teminology) of such structures. For example using sand for an idealized model of such systems we expect by pouring the same amount of send on a table to obtain every time a heap of different shape (and space configuration of grains)but pretty much of the same properties and averaged dimensions. It also means that formation of monolayer of grains (having the lowest potential energy in the gravitational field) can be estimated as event of very low probability under such conditions as well as will be low probability to obtain extremely high and slim heaps. More detailed analyses will confirm selfsimilarity and scaling of properties in such systems as we will see later. Still it arises several fundamental issues: Why equilibrium- based description should apply to these non- equilibrium systems? Moreover, thermal equilibration is not possible because grains are too heavy for thermal motions. How than to formulate a phase space that enable the treatment of such jammed particle systems in the systematic ways of statistical mechanics. Those difficulties are one of the main reasons why granular matter as one of the most frequently manipulated materials in human trade history is so little elaborated in exact way. The first break- through has been maid by S.E. Edwards and his group at Cambridge [7]. In short, they used free volume in the cluster of particles as the conserved quantity that takes over the place that has energy in the Boltzmann statistics. Than for the heap of send in a box of volume $V$ with $N$ send grains under given conditions as described above, there is $\Omega(N, V)$ distinct mechanically stable states. Now in analogy to Boltzmann definition of temperature can be written

$$
\frac{1}{X}=\left(\frac{\partial \ln \Omega}{\partial V}\right)_{N}
$$

Here, $X$ is compactivity of the system that describes spreading of particles inside the cluster. As the particles are more spread around (inside the cluster!) the packing is looser and there is more free volume in the cluster what indicates less interactions and relatively weaker interaction forces in that state of the cluster $[7,8]$. That makes in Edwards's theory the link to the energy approach of the classical statistical mechanics. Indeed, if the energy is broth to the pile of sand e.g. vibrating it, the packing of reproducible densities will be obtained, as experiments indicate $[1,8-19]$. It enables proposal of steady state distribution of configurations providing link to equilibrium based distributions in statistical mechanics [15-19]. In this new formalism the partition function for the cluster of grains, considered as canonical ensemble, can be written

$$
Z_{W}=\int \theta\left(q_{n}\right) \mathrm{e}^{\frac{-W_{d}\left(q_{n}\right)}{\lambda X}} \prod_{n}\left\{\mathrm{~d} q_{n}\right\}
$$

where $q_{n}$ are degrees of freedom that comprise the phase space and $\theta\left(q_{n}\right)$ is probability density of configuration $\left\{q_{n}\right\}$ at particular point of this space, $W_{d}$ is called volume function and represents the analogue of Hamiltonian in classical statistical mechanics, $X$ is compactivity and $\lambda$ is the constant regulating dimensions for the exponent. For systems of living cells that are of interest here we can in a similar way define the steady state distribution of cluster configurations [8]. Some of cell types have rather stiff outer layer giving them permanent shape as particles in Edwards's terms and connect each to other with receptor -ligand -complex producing kind of fixed contact points also in terms of Edwards's model. Moreover, cells in cluster make fluctuation- movements what changes their orientation in space and in that way change the free volume distribution in the cluster as well. It follows that we can describe the steady states of a cluster by equation analogue to Eq. (2). According to classical definition [9] free volume of the particle $w_{f r}$ is the difference between the volume available to the particle for its oscillations inside a cluster and a volume of the particle itself. For living cells there is some maximal free volume $w_{\max }$ over which cell will loose its contact with the cluster and some minimal free volume $w_{\text {min }}$ that cell requires for its normal life [8]. According to that and Eq. (2) we can write the partition function of a cluster of $N$ cells

$$
Z_{c l}=\int \theta(w) \prod_{i}^{N}\left(\mathrm{e}^{\frac{-w_{i}}{\lambda X}} \mathrm{~d} w_{i}\right) .
$$

In the same way as for particles in the Boltzmann lattice, exchanging energy states, and here for anchored cells in the lattice exchanging the free volume around them we can write

$$
Z_{c l}=\left[\theta \int_{w_{\min }}^{w_{\max }} \mathrm{e}^{-w /(\lambda X)} \mathrm{d} w\right]^{N}=z^{N}
$$

where $z$ is the volumetric partition function of the cell in a cluster.

Still, there are some essential differences in formulations of Edwards's model and the one of interest here: (1) The large number of cell types changes shape due to the soft membrane and elastic surface layers, so Edwards's conditions for free volume estimation can not be applied strictly [8-19].

(2) Also because cells generate elastic forces to its environment, especially to overcome resistance of the surrounding gels to cluster growth. 
(3) The interactions between cells are much more complex than the friction between send grains in Edwards's model. To describe those phenomena we need to formulate Hamiltonian for elastic - and binding- energies between cells in cluster.

Since Bell's seminal paper [20] numerous investigations have been published considering cell-cell interaction and formulating different equations for interaction relations but all consider cell- cell interactions as kinetic process that can be described by reversible equation

$$
R_{e c}+L_{i g} \underset{k_{r}}{\stackrel{k_{f}}{\leftrightarrows}} R_{e c} L_{i g}
$$

where $R_{e c}$ are receptor molecules and $L_{i g}$ are ligands, $k_{f}$ is kinetic constant of receptor -ligand complex formation and $k_{f}$ kinetic constant for its break. According to Bell: $k_{r}=k_{0} \exp \left(r_{0} F_{b} / k_{\mathrm{B}} T\right)$ where $k_{B}$ is Boltzmann constant $T$ temperature $r_{0}$ is critical distance and $F_{b}$ the bond strength. With progress of experimental measurements at nano-level including different peeling tests and especially dynamic force spectroscopy [21-23] last years are available good experimental data for different systems what opens new possibilities for quantitative treatment and theoretical predictions. But, description of force transfer and binding connections between cells requires still further elaboration. In that purpose and starting from principles and properties of polymer networks [9] applied to the network interpenetrating the cluster as described above, we write Hamiltonian of cell- cell interaction contact in the form

$$
H_{c}(r)=H_{e l}(r)+H_{b i n d}(r)
$$

Dynamics of network segments and dangling chains can be described as dynamics of bead and spring oscillators by harmonic potentials. But one cell $i$ is under influence of $j$ cells in contact with it. For elastic energy of such system we can write

$$
H_{e l}(r)_{i}=\sum_{j} \frac{a_{j}^{2}}{2} k_{F b}\left(r_{j}-r_{b}\right)^{2}
$$

where $k_{F b}$ is stiffnes constant per unit of cell surface and $a$ characteristic dimension of the $j$ contact, $r_{j}$ is distance between contact patches of referent cell an cell $j$ and $r_{b}$ is the rest length. In the similar way oscilations around critical distance $r_{b c}$ of $R_{e c}$ binding with $L_{i g}$ can be discrebed by

$$
H_{b i n d}(r)_{i}=\sum_{j_{R}, j_{L}} \frac{a_{j}^{2}}{2} k_{f, b i n d}\left(r_{j}-r_{c, b}\right)^{2}
$$

But, the formation of cell-cell interaction complex depends also on the space configuration of cell-cell surface pairs in interaction and further receptor -ligand pairs at those surfaces. So we have to formulate partition function of contact probabilities between cells in cluster

$$
Z_{\text {Cint }}=\sum_{i} \prod_{i} \sum_{j} \theta \mathrm{e}^{\frac{\sum_{i, j} H_{i, j}}{k_{\mathrm{B}} T}}
$$

As values for pagebreak reference energy $k_{B} T$ in Eq. (7) can be used $4.2 \mathrm{pN} n \mathrm{~nm}$ at physiological temperature 310 $\mathrm{K}$, based on data from nano- measurements but, gener- ally it is rather difficult to estimate $\mathrm{Z}_{\text {Cint }}$ numerically. Also, the Hamiltonian in the exponent can be transformed rearranging Eq. (6a) and Eq. (6b) to united potential but keeping in mind that frequencies and amplitudes of two kinds of oscillations described can be quite different. In that way we come to the potential

$$
U_{e f}=K_{F b}\left(\tau, r_{c}, E_{0}\right)\left(r_{i, j}-r_{c, b}\right)^{2}
$$

The new stiffness parameter $K_{F b}$ is now function of time and amplitudes but similar united parameters are formulated by some other authors and can be used at relevant time scales with $K_{F b}$ practically constant as a number of experiment indicate and will be discussed later [21]. Anyway, we can suppose that the same potential holds for all contacts between the same cells in a cluster (but the interaction forces differ depending of the distance). Then stress tensor in a cluster of volume $V_{c c}$ with $N$ cells reads

$$
\sigma_{c c}=\frac{1}{2 V_{c c}} \sum_{i, j} \boldsymbol{L}_{i, j} \boldsymbol{F}_{i, j}
$$

where $\boldsymbol{F}_{i j}$ is interaction force between cells $i$ and $j$ and $\boldsymbol{L}_{i, j}=\boldsymbol{l}_{i, j}-\boldsymbol{l}_{j, i}$ is connection vector between cells with $\boldsymbol{l}_{i j}$ contact vectors from relevant center of corresponding cell in a pair to the contact. The stress $\boldsymbol{\sigma}_{c c}$ can be related to the free volume of the cluster in the sense that interactions forces $\boldsymbol{F}_{i, j}$ keeping in balance a particular cluster configuration in space, generate at the same time interstitial space e.g. voids and holes in the cluster or free volume. Let be the measure of each such void $\nu$ surrounding cell $i$ some vector $\boldsymbol{g}_{i, j}$ and call it generated void vector and let the contact pint separate two voids $\nu(h, j)$ and $\nu(j, o)$ around the cell $i$. Based on previous statements we can write

$$
\boldsymbol{g}_{i,(h, j)}-\boldsymbol{g}_{i(j, o)}=\boldsymbol{F}_{i, j} .
$$

The possibility to establish univocal correspondence between generated void vectors, measuring free volume and the stress tensor of the cluster, can be easily shown in the case of stable position of a cell $i$ in contact with 3 cells at the flat surface. It follows that contact points can be designated by $j=1,2,3$ and voids in-between by $\nu=(1,2)$, $(2,3),(3,1)$. For the cell $i$ we write the balance of interaction forces

$$
\boldsymbol{F}_{i, 1}+\boldsymbol{F}_{1,2}+\boldsymbol{F}_{1,3}=0 .
$$

Let the measure of void $\nu=(1,2)$ be $\boldsymbol{g}_{i(1,2)}=\boldsymbol{g}_{0}$. Based on Eq. (9) we can write

$$
\begin{aligned}
& \boldsymbol{g}_{i(3,1)}-\boldsymbol{g}_{i(1,2)}=-\boldsymbol{F}_{i, 1}, \\
& \boldsymbol{g}_{i(2,3)}-\boldsymbol{g}_{i(3,1)}=-\boldsymbol{F}_{i, 3}, \\
& \boldsymbol{g}_{i(1,2)}-\boldsymbol{g}_{i(2,3)}=-\boldsymbol{F}_{i, 2} .
\end{aligned}
$$

Substituting Eq. (12a), (12b) and Eq. (11) into Eq. (12c) we obtain always the same measure:

$$
\begin{aligned}
& \boldsymbol{g}_{0}=\boldsymbol{g}_{i(1,2)}=\boldsymbol{g}_{i(2,3)}-\boldsymbol{F}_{i, 3}=\boldsymbol{g}_{i(1,2)}-\boldsymbol{F}_{i, 1} \\
& -\boldsymbol{F}_{i, 2}-\boldsymbol{F}_{i, 3}=\boldsymbol{g}_{i(1,2)} .
\end{aligned}
$$

The identity Eq. (13) can be extended to larger number of particles and always in the same way calculate moment of forces laid upon cell $i$ defined as: 


$$
\begin{aligned}
\sigma_{i} & =\sum_{j} \boldsymbol{l}_{i, j} \boldsymbol{F}_{i, j}=\sum_{j} \boldsymbol{l}_{i, j}\left(\boldsymbol{g}_{i(i, j)}-\boldsymbol{g}_{i(j, i)}\right) \\
& =\sum_{j}\left(\boldsymbol{l}_{i, j}-\boldsymbol{l}_{j, i}\right) \boldsymbol{g}_{i, \nu}=\sum_{\nu} \boldsymbol{s}_{i, v} \boldsymbol{g}_{i, v}
\end{aligned}
$$

Vectors $\boldsymbol{s}_{i, v}$ we call segment vectors connecting contact points of $i$ and neighboring cells to be much easier to determine then vectors $\boldsymbol{l}_{i, j}$ and $\boldsymbol{L}_{i, j}$. It is in fact universally defined parameter for the whole cluster. Segment vectors extend through the whole cluster making continual connectivity network between all contact complexes. It is at the same time topological network and interaction network as we will see, directly related to free volume by products with void generating vectors $g_{i v}$ as can be seen from Eq. (14). Now, based on the same principle, the procedure can be extended to define interaction force moment tensor for the whole cluster $\boldsymbol{\Phi}_{C C}$ :

$$
\begin{aligned}
\Phi_{C C} & =V_{C C} \sigma=\frac{1}{2} \sum_{i, j} \boldsymbol{L}_{i, j} \boldsymbol{F}_{i, j} \\
= & \frac{1}{2} \sum_{i, j}\left(\boldsymbol{l}_{i, j}-\boldsymbol{l}_{j, i}\right) \boldsymbol{F}_{i, j}=\frac{1}{2} \sum_{i, j}\left(\boldsymbol{l}_{i, j} \boldsymbol{F}_{i, j}+\boldsymbol{l}_{j, i} \boldsymbol{F}_{j, i}\right) \\
= & \sum_{i, j} \boldsymbol{l}_{i, j} \boldsymbol{F}_{i, j}=\sum_{i} \sigma_{i}=\sum_{i} \sum_{v} \boldsymbol{s}_{i, v} \boldsymbol{g}_{i, v} .
\end{aligned}
$$

Several authors related in a similar way force moment tensor with free volume measuring parameters for clusters of micro particles and grains [10-20] but not for living cells. Some of constellations around voids for the particles considered were much more complicated but from all the cases can be derived conclusion that $\boldsymbol{\Phi}_{C C}$ is additive over subsystems of cluster particles closing the voids and that $\boldsymbol{\Phi}_{C C}$ is conserved for such a system in a box with fixed boundary conditions. It follows that such systems satisfy basic requirements for treatment as statistical mechanical ensembles and can be called stress ensemble. In Edwards's terms for the number of states $\Omega\left(\Phi_{C C}\right)$ we can write angoricity in the form

$$
\alpha=\left(\frac{\partial \ln \Omega}{\partial \Phi_{C C}}\right)_{N}
$$

Considering cluster environment as large enough reservoir in energy balance with the cluster probability for state $h$ is

$$
P\left(\Phi_{c c}\right)_{\kappa} \propto \Omega \mathrm{e}_{\kappa}^{\left(-\alpha \Phi_{c c}\right)}
$$

what also requires extensive insight for elaboration. But for the cell cluster, we should be aware of coupling between elastic responses and contact coupling, binding and rebinding contacts as described by Eq. (5)-(8). Moreover, experiments indicate that cells in clusters keep some permanent and unified tension upon substrate, probably as cooperative activity of cytoskeleton and cell adhesion complexes. Indeed, some very interesting experimental results of several groups of authors [21-23] show that size of mature focal adhesion complex can adduce itself keeping constant pressure even for cells that are not under constrains from environment. In that case the tension per unit of area (stress) is $5.5 \mathrm{k} \mathrm{Pa}$ and remarkable constant among different cell types $[25,26]$. It provides background for mean field approach considering cluster states under isotropic compression, in balance with environment and substituting tensor $\boldsymbol{\Phi}_{C C}$ with scalar, simply with $p V_{c c}$ where $p$ is pressure in the cluster and $V_{c c}$ is its volume:

$$
\varphi=p V_{C C}=\frac{1}{2} \sum_{i, j} L_{i, j} F_{i, j}
$$

Then Eq. (17) reads

$$
P(\varphi)_{\kappa} \propto \mathrm{e}^{-\alpha \varphi_{\kappa}}
$$

and partition function of the cluster is

$$
Z_{C C}(\alpha)=\sum_{\kappa} \mathrm{e}^{-\alpha \varphi_{\kappa}}
$$

Keeping in mind that cell under conditions described can change their shape but not the volume, under isotropic deformation field we can eliminate variable $L_{i, j}$ from Eq. (18)-(20) and write partition function just in terms of $F_{i, j}$, because of one to one relation between $\left\{L_{i, j}\right\}$ and $\left\{F_{i, j}\right\}$ sets. Denoting average volume of a cell in the cluster by $w_{c, a v r}$ we can instead $\mathrm{L}$ use equivalent distance $\left(6 w_{c, a v r} \pi\right)^{1 / 3}$ and the partition function reeds

$$
Z_{C C}(\alpha)=\sum_{\{F\}} \mathrm{e}^{\left.-\alpha \frac{6 w_{c, a v r}}{\pi}\right)^{\frac{1}{3}} \sum_{i, j} F_{i, j}}
$$

Analogue to Eq. (4), voids we can decuple in the first approximation of interaction pairs and obtain

$$
\begin{gathered}
Z_{C C}(\alpha)=\prod_{i, j}^{\frac{N \xi_{0}}{2}} \int_{0}^{\infty} d F \mathrm{e}^{-\alpha(6 w / \pi) F} \\
=\left(\frac{\pi^{\frac{1}{3}}}{\alpha\left(6 w_{c, a v r}\right)^{\frac{1}{3}}}\right)^{\frac{N \xi_{0}}{2}}
\end{gathered}
$$

where $\xi_{0}$ is number of contacts per cell. It is equal to number of degrees o0f freedom of a cell in a cluster [8]. From Eq. (18)-(22) we obtain the average value of $\phi$.

$$
\left\langle p V_{C C}\right\rangle=\frac{-\partial \ln Z_{C C}}{\partial \alpha}
$$

From Eq. (22)-(23) and denoting number density of cells by $\left(N / V_{C C}\right)=\rho_{c c}$, we obtain equation of state for cluster

$$
p \frac{2 \alpha}{\xi_{0}}=\rho_{c c}
$$

Considering derivatives with time in the sense of cluster grow with increase of cell number $N$ under conditions described, we obtain

$$
\boldsymbol{w}_{\boldsymbol{p}} \frac{2 \alpha}{\xi_{0}}=W_{c c c}
$$

where $\mathrm{w}_{p}$ is the rate of pressure increase and $W_{c c c}=$ $\left(\rho-\rho_{0}\right) /\left(t-t_{0}\right)$ we call the rate of cumulative increase of cluster density or rate of cluster density increase. The increase of number of cells in a cluster due to proliferation, we can describe using effective rate constant $k_{c, e f}$ :

$$
\frac{\mathrm{d} N}{\mathrm{~d} t}=k_{c, e f} N
$$

Integrating Eq. (26) from $N_{0}$ to $N$ we obtain for the increase of cluster density, in particular period of time $t-t_{0}$ 
starting with density $\rho_{0}$ :

$$
\begin{aligned}
& W_{c c c}=\frac{1}{t-t_{0}}\left[\frac{N}{V_{0}-N w_{c, a v r}}-\frac{N_{0}}{V_{0}}\right] \\
& =\frac{1}{t-t_{0}}\left[\frac{N_{0} \mathrm{e}^{k_{c, e f} t}}{V_{0}-N_{0} \mathrm{e}^{k_{c, e f} t}}-\frac{N_{0}}{V_{0}}\right] \\
& \approx \frac{\mathrm{e}^{k_{c, e f} t}-1}{t-t_{0}} \frac{N_{0}}{V_{0}}
\end{aligned}
$$

The approximation is valid if space available in time $t$ for a cell is much larger than cell's average size (in the sense that we discussed with Eq. (4)). Finally, integrating Eq. (25) over some period of time from $t_{0}$ to $t$ we obtain the increase of pressure in the cluster in the form

$$
\begin{aligned}
& \frac{2 \alpha}{\xi_{0}}\left(p-p_{0}\right)=\rho_{0}\left(\frac{k_{c, e f} t}{1 \cdot 1 !}+\frac{\left(k_{c, e f} t\right)^{2}}{2 \cdot 2 !}\right. \\
& \left.+\frac{\left(k_{c, e f} t\right)^{3}}{3 \cdot 3 !}+\cdots\right)
\end{aligned}
$$

In previous equations we consider $\alpha$ and $\xi_{0}$ constant, that means the same organization and degrees of freedom in the cluster. But with increase of number of cells in the cluster its state approaches to singularity as described in Eq. (27).Cells in the cluster can overcome such situation ( to some extent also depending on organization of the cluster) by adducing their positions, to decrease in that way the average volume they use in the cluster. But, the change of $\mathrm{w}_{c, a v r}$ means also the changes of $\alpha$ and $\xi_{0}$. This is directly related to Eq. (23) in our previous paper on jammed states in living cell clusters [8]. In this way our theory covers behavior of cell clusters from the very beginning of its organization till stop of growth due to increase of density and decrease of free volume in the cluster indicated by some critical value of volume per cell $w_{\text {min }}$.

\section{Results and discussion}

The theory proposed in previous section relates rheological properties of a single cell (with data in remarkable progress of quality and accumulation last years due to measurements on nano-level) with properties of cellclusters, been of high importance for understanding development and behavior of cell cultures and tissues [2035 . In the theory are also considered very serious difficulties in quantitative description of cell cluster behavior using micromechanics and rheology. In literature it is explained by changes of cell interactions and relations in space with proliferation, grow and self-assembling of clusters $[1-4,32-35]$. But, in derived relations of the theory proposed a significant number of such difficulties is eliminated by appropriate averaging over cluster sub -systems in space and time. In that way the method proposed is incorporating into modern approach in physics of jammed state where accumulated mechanical energy of subsystems, appropriately averaged, can be added according to the principles of adding energy in thermodynamics [6-12]. For example $\sum \boldsymbol{\sigma}_{i}$ in Eq. (15) can be extended substituting segment vectors by $\boldsymbol{s}_{i, j}$ that are bond to cell, by $\boldsymbol{s}_{i, j}=\boldsymbol{q}_{i, v}+\boldsymbol{h}_{i, v}$ that gives now sum of vectors related to voids :

$$
\boldsymbol{\Phi}_{\boldsymbol{s}}=\sum_{i} \boldsymbol{\sigma}_{i}=\sum_{i \in S} \sum_{v}\left(\boldsymbol{q}_{i, v}+\boldsymbol{h}_{i, v}\right) \boldsymbol{g}_{i, v}
$$

So, contributions from the interior boundaries in area $S$ cancel pair -wise due to adding the terms going contra clockwise around the boundary of cell $i$ and the terms from going contra -clockwise around its neighboring cell $j$ through the voids common to grains $i$ and $j$.In such a way we obtain contributions for expansion of cluster and changes of density from generated void variables at the boundaries to insert in Eq. (15). One of parameters that incorporate such coupling is rate of cluster density increase $W_{c c c}$ presented in Fig. 1.

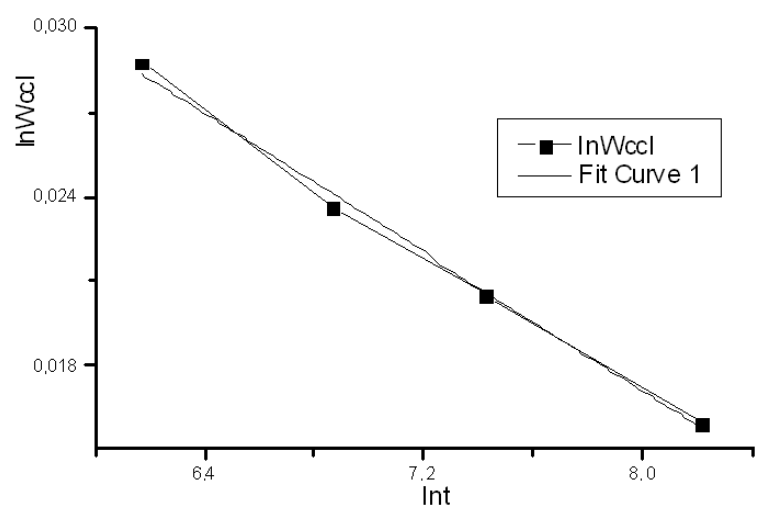

Fig. 1. Scaling of rate of cluster density increase $W_{c c c}$ with time $t$ [hours].

The change of cluster density with time due to the increase of number of cells $\mathrm{N}$ according to those principles and the model derived from our theory can scale in Eq. (27) in two ways :(a)If there is a lot of space for expansion of cluster $\ln W_{c c c}$ will be proportional to $k_{c, e f} t$ (b) if the space available for cell in the cluster is close to $w_{c, a v}$ than $\ln W_{c c c} \sim-\ln \left[w_{c}(t)-w_{c, a v r}\right]-\ln t$. From experiments on yeast cells in alginate beads described in our previous papers [24-30] and Eq. (27) we calculated results presented in Fig. 1. The results indicate cumulative increase of cluster density and shortage of space, as expected. Moreover, incorporation of Eq. (8) in calculations provides possibilities to translate the system parameters into topology -geometry relations scaling with time.

\section{Conclusions}

Interactions of living cell with neighboring cells in a cluster arising from interactions in cell cultures, tissues and encapsulating polymer beads or micro-capsules are of essential importance for their life, realization of different targets of their cultivation. In spite to that, a number of issues as early stop of colony growth, early decrease of performances and understanding of some transitions of cluster structure [8] related to criticality issues, require further investigations. But, standard protocols of 
cell colony cultivation and characterization do not provide adequate data for many of such investigations. In the theoretical part of this work, we proposed equations relating standard parameters from cell colony characterization with basic interactions influencing grow and organization of cell clusters. Moreover, the model provides possibilities for incorporating data from nano-rheological single -cell behavior measurements giving promising data of high interest, last years.

\section{Acknowledgment}

This research was funded by grant (\# 46010) from the Ministry of Science and Technological Development, Republic of Serbia.

\section{References}

[1] B. Alberts, D. Bray, J. Lewis, M. Raff, K. Roberts, J.P. Watson, Molecular Biology of the Cell, Garland, New York 2004.

[2] S. Monte, F. Ovidio, S. Dane, P. Sorensen, PNAS 104,18 377 (2007).

[3] A.F. Taylor, M.R. Tinsly, F. Wang, Z. Huang, K.Schwalter, Science 323, 614 (2009).

[4] D. Rakovic, Integrative Biophysics Quantum Medicine, Quantum Holographic Informatics, IASC-IEFPP, Belgrade 2009.

[5] G.T. Gregor, K. Fujimoto, N. Mosoki, S. Sawai, Science, 328, 1021(2010).

[6] P.G. deGennes, Rev. Mod. Phys. 71, S374(1999).

[7] S.F. Edwards, J. Stat. Physics 116, 29 (2004).

[8] M.B. Plavsic, I. Pajic-Lijakovic, M.M. Plavsic, Int. J. Modern Phys. 24, 813(2009).

[9] P.J. Flory, Principles of Polymer Science (Cornel Univ. Press., New York 1971).

[10] H.A. Makse, J. Kuchan, Nature 415, 614 (2002).

[11] R.C. Ball, R. Blumenfeld, Phys. Rev. Lett. 88, 115505 (2002).

[12] R. Blumenfeld, S.F. Edwards, J.Phys. Chem.B, 113, 3981 (2009)

[13] S. Henkes, C.S. O'Hern, B. Chakraborty, Phys. Rev. Lett. 99, 038002 (2007).

[14] C. Song, P. Wang, H. Makse, Nature 453, 629 (2008).

[15] S.F. Edwards, Phys. A 353, 114 (2005).
[16] S.F. Edwards, J. Phys. A: Math. Theor. 41, 3240 (2008).

[17] J. Brujic, P. Wang, C. Song, D. Johnson, O. Sindt, H. Makse, Phys. Rev. Lett. 95, 128001(2005).

[18] E. Nowak, J. Knight, E. Ben-Naim, H. Jaeger, S. Nagel, Phys. Rev. E 57, 1971 (1998).

[19] J. Bray, A. Prados, Phys. Rev. E 68 ,051302 (2003).

[20] G.I. Bell, Science, 200, 618 (1978).

[21] E.A. Evans, D.A. Chalderwood, Science, 316, 1148 (2007).

[22] D. Riveline, E. Zamairn, N. Balaban, U. Schwarz, T. Ishizaki, J. Cell Biology 153, 1175 (2001).

[23] N.Q. Balaban, U. Schwarz, D. Riveline, P. Goichberg, G. Tzur, Nature Cell Biology 3, 466 (2001).

[24] I. Pajic-Lijakovic, M. Plavsic, V. Nedovic, B. Bugarski, J.Microencap. 24, 420 (2007).

[25] I. Pajic-Lijakovic, D. Bugarski, M. Plavsic, B. Bugarski, Process Biochemistry 42,167 (2007).

[26] I. Pajic-Lijakovic, M. Plavsic, B. Bugarski, V. Nedovic, J. Biotech. 129, 446 (2007).

[27] I. Pajic-Lijakovic, M. Plavsic, V. Nedovic, B. Bugarski, Minerva Bioteh. 20, 99 (2008).

[28] I. Pajic-Lijakovic, B. Bugarski, V. Nedovic, M. Plavsic, Minerva Bioteh. 17, 245 (2005).

[29] I. Pajic-Lijakovic, V. Ilic, B. Bugarski, M. Plavsic, Europ. Biophys. J. 39, 789 (2010).

[30] M.B. Plavsic, I. Pajic-Lijakovic, N. Lazic, B. Bugarski, P. Putanov, Materials Manufac. Proc. 24, 1190 (2009).

[31] M.B. Plavsic, I. Pajic-Lijakovic, P. Putanov, Ch. 19, Chain Conformational Statistics, Mechanical Properties of Elastomer Blends in: New polymer materials, Eds. L. Korugic-Karaz, W. Mac Knight, E. Martuscelli, ACS edition, Oxford Univ. Press, Oxford 2005.

[32] M.B. Plavsic, I. Pajic-Lijakovic, B. Bugarski, J. Budinski-Simendic, V. Nedovic, P. Putanov, Ch. 14, Pseudo-Blend Model of Hydrogel Immobilized Living Cells in: Contemporary Science of Polymeric Materials, Ed. L. Korugic-Karasz, ACS Books edition, Washington 2010.

[33] J.P. Hsu, A. Spasic, Interfacial Electroviscoelasticity, Electrophoresis, CRC Press, New York 2010.

[34] G. Chang, J. Tse, R.K. Jain, L.L. Munn, PloS ONE 4 e4632 (2009).

[35] J. Guck, F. Lautenscklager, S. Pasche, M. Beil Int. Biol., pub. on line 27 Sep. 2010, http://pubs.rsc.org|doi:10.1039/COIB00050G. 